\title{
Aqueous Lubrication with Poly(Ethylene Glycol) Brushes
}

\author{
Nicholas D. Spencer \\ Laboratory for Surface Science and Technology, Department of Materials, ETH Zurich \\ Vladimir-Prelog-Weg 5, 8093 Zurich, Switzerland \\ Corresponding author: nspencer@ethz.ch
}

\begin{abstract}
( Manuscript received 30 April 2014; accepted 27 July 2014; published 15 October 2014 )
(Presented at the International Tribology Forum Tokyo 2013 at the Katsushika Campus, Tokyo University of Science, October 2013 )
\end{abstract}

\begin{abstract}
Hydrophilic polymer brushes constitute a man-made approach to imitating nature's lubrication mechanisms. A polymer that has been frequently used to explore such systems is poly(ethylene glycol) (PEG). While PEG may not be the ideal solution for water-lubricated tribosystems for a number of technical reasons, this well-characterized polymer has served as an extremely useful model for the development of other polymer-brush-based lubricant approaches. This review covers the history of PEG brushes used as aqueous lubricants, including the large body of work on electrostatically attached PEG brushes, and ends with a discussion of current and future research directions that build upon the knowledge gained over a decade and a half of PEG-brush research in tribology.
\end{abstract}

Keywords: aqueous lubrication, PEG, polymer brush, poly(ethylene glycol), poly(ethylene oxide)

\section{Introduction}

Aqueous lubrication is a seductive idea. It has the potential to provide a solution that is environmentally compatible and sustainable, is highly effective for thermal management, and lowers lubricant-transport costs, since the end-user can simply blend a concentrate with water. Aqueous lubrication is also nature's approach [1], and natural lubricants have served as an inspiration to those working in this field over the last couple of decades $[2,3]$. With the exception of its use in winter sports in the form of ice near $0^{\circ} \mathrm{C}$, water in a pure state is a poor lubricant. There are several reasons for this. Firstly it is intrinsically of low viscosity $\left(\approx 1 \times 10^{-3} \mathrm{~Pa}\right.$ at room temperature), while the viscosity of oils is typically several orders of magnitude higher. Secondly, while the viscosity of oil increases exponentially with pressure (a critical factor in the elastohydrodynamic lubrication mechanism occurring in non-conformal contacts, such as gears or rolling-element bearings [4]), the pressure dependence of water's viscosity is negligible [5]. Thirdly, water has no boundary-lubrication capability. Nature solves these problems by lubricating only soft contacts, and also by the use of lubricious glycoprotein-based additives such as mucins [6], which coat the sliding surfaces with a highly hydrated array of sugar chains.

When polymers are fixed by their chain-ends to a surface, and the whole system is immersed in a good solvent for the polymer, the chains stretch out into the solvent, so as to minimize the chain-chain interaction energy. The resulting structure is known as a polymer brush [7]. Polymer brushes, used in lubrication, are man's attempt to mimic the structure of natural molecules such as mucin. Much of the pioneering work in this area has been carried out by Klein and his group [8-10], his initial studies focusing on polystyrene chains surrounded by toluene. Klein suggested that the brushes lubricated well, due to the presence of entropic forces that held the solid surfaces apart, while maintaining a fluid (low-shear-strength) film between them. He went on to suggest that this may be the origin of the low friction encountered in natural lubrication systems, such as synovial joints. While Klein's model still appears to be a very plausible explanation for the lubricity of brushes, the lubrication mechanisms at work between glycoproteins now appear to be somewhat more complicated [11].

In order to use polymer brushes for aqueous lubrication, a polymer must be employed for which water is a good solvent. One readily available and much studied such water-soluble polymer is poly(ethylene glycol) (PEG) (Fig. 1). PEG is uncharged, non-toxic, and used in a variety of applications ranging from pharmaceuticals to wood preservation. PEG brushes have been extensively used for inhibiting protein adsorption onto surfaces [12].

While PEG was a clear choice as a starting point in aqueous lubrication with polymer brushes, it has served more as a very useful model system rather than one with major potential for widespread applications. There are a 


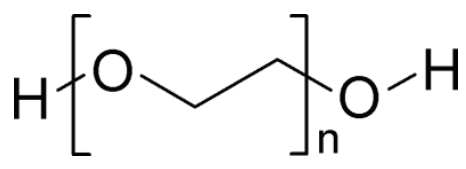

Fig. 1 Poly(ethylene glycol), also known as poly (ethylene oxide) or PEG

number of reasons for this, including its susceptibility to oxidation [13] and its lower critical solution temperature [14], which can be well below $100^{\circ} \mathrm{C}$, restricting its use to moderate temperatures. Nevertheless the insights gained from a decade and a half of PEG-brush-based aqueous lubrication have paved the way for the exploration of further water-based systems, which may have greater applicability.

\section{Early studies on aqueous lubrication with PEG brushes}

Raviv et al. [15] attached PEG chains (3.4 kDa) to mica surfaces, for a surface-forces apparatus study, by means of end-functionalizing the PEG with a trimethylammonium group that presumably attached via the ion-exchangeable cation sites on the mica surface. The mean chain-chain spacing on the surface was on the order of $2 \mathrm{~nm}$. At low compressions, no frictional forces could be detected, but as the compression was increased, the friction increased markedly. This was attributed to the effects of chain interpenetration leading to viscous dissipation and bridging effects. The Klein group also investigated a high-molecular-weight PEG (150 kDa), attached to the mica surfaces purely by ion-ligand interactions [16]. In this case, as compression was increased, the measured shear values reached a saturation value, attributed to a shift from a polymer-polymer slip plane to one between the polymer and the substrate.

Block copolymers of PEG and poly(propylene oxide) (PPO) have been used as aqueous lubricants for hydrophobic surfaces. This is possible because although PEG is hydrophilic, PPO is quite hydrophobic, and therefore can act as a surface anchor to a hydrophobic surface, when linked to PEG in a diblock or triblock copolymer, for example. Such copolymers have many industrial applications, such as emulsion stabilization or as detergents. They are commercially available in many architectures, and are known as poloxamers or Pluronics ${ }^{\circledR}$. Lee et al. investigated the effect of PEO-PPO-PEO architecture on the aqueous lubrication of a model hydrophobic surface, poly(dimethylsiloxane) (PDMS) [17]. The molecular weight of the central PPO block appeared to determine the propensity of the PEO-PPO-PEO tribock to adsorb from aqueous solutions onto the PDMS surface. Once adsorbed, the Pluronic $\left(\mathrm{EO}_{37} \mathrm{PO}_{56} \mathrm{EO}_{37}\right.$, trade name P105) appeared to rid the system of the strong hydrophobic adhesion that is characteristic of PDMS-PDMS contact in water,

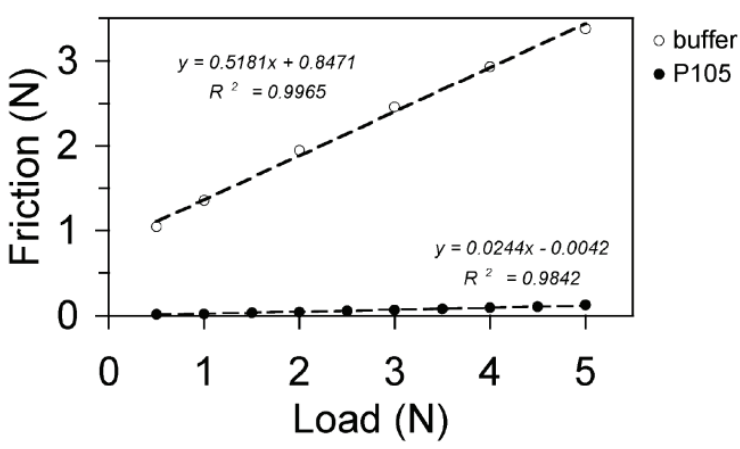

Fig. 2 Friction vs load plot for the self-mated sliding of PDMS lubricated by buffer alone $(\circ)$ and $\mathrm{EO}_{37} \mathrm{PO}_{56} \mathrm{EO}_{37}$ (P105)-containing buffer solution $(\bullet)$. The sliding speed was $0.005 \mathrm{~m} / \mathrm{s}$. Reprinted from [17] with kind permission from Macromolecules. Copyright 2004, the American Chemical Society

replacing it by a bound aqueous layer held within the PEG chains, that drastically reduced the measured friction (Fig. 2).

Li et al. [18] studied the interaction and aqueous lubrication properties of $\mathrm{EO}_{19} \mathrm{PO}_{29} \mathrm{EO}_{19}$ with surfaces of polyethylene, polypropylene (both hydrophobic) and cellulose (hydrophilic). They used a combination of lateral force microscopy and XPS, to measure friction and surface adsorption, respectively. As might be expected, the triblock lubricated PE and PP, but not cellulose. More surprisingly, it appeared to be more firmly affixed to the PE than the PP, from which it could be readily removed by washing in water.

\section{Approaches to PEG brush lubrication, by means of various tethering methods}

3.1. Poly(L-lysine)-g-poly(ethylene glycol) (PLL-g-PEG)

In the previous section, an approach was discussed whereby a hydrophobic block on a block copolymer can be used to anchor a PEG chain onto a hydrophobic surface. Many natural and inorganic surfaces bear charges, and thus the idea of a PEG-polyelectrolyte block copolymer came about. A particularly efficient way of doing this was invented in 1998 by Elbert and Hubbell [19], as a means of modifying the surfaces of cells with PEG, in order to influence their adhesive properties. The approach involves the synthesis of a comb polymer, known as poly(L-lysine)-g-poly(ethylene glycol) (PLL-g-PEG) (Fig. 3a), consisting of a charged, poly(L-lysine) backbone and PEG side chains. Since only a fraction of the free amine groups on the lysine side chains are functionalized with the PEG, the remainder, which acquire a positive charge in neutral aqueous environments, serve as electrostatic anchors onto negatively charged surfaces, such as oxides [20] (Fig. 
a)

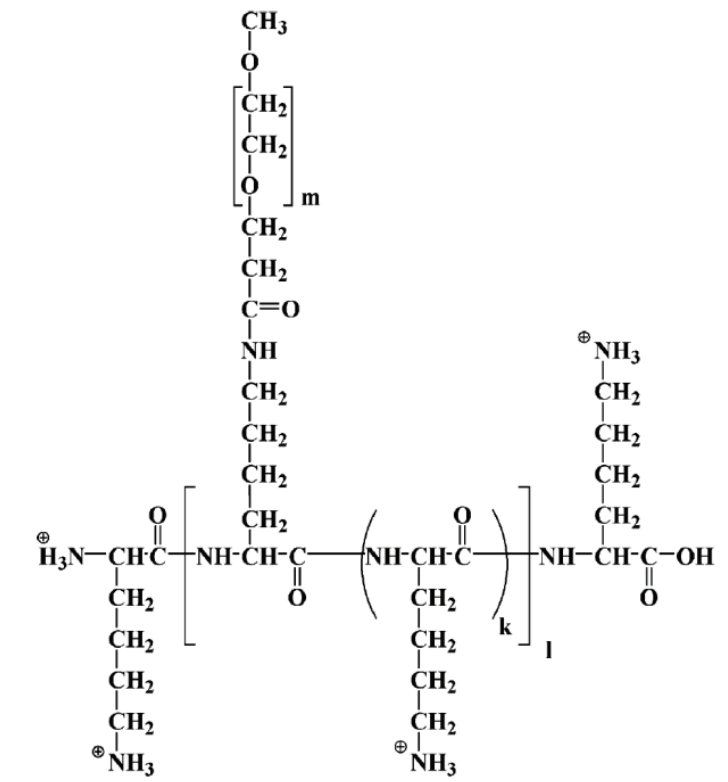

b)

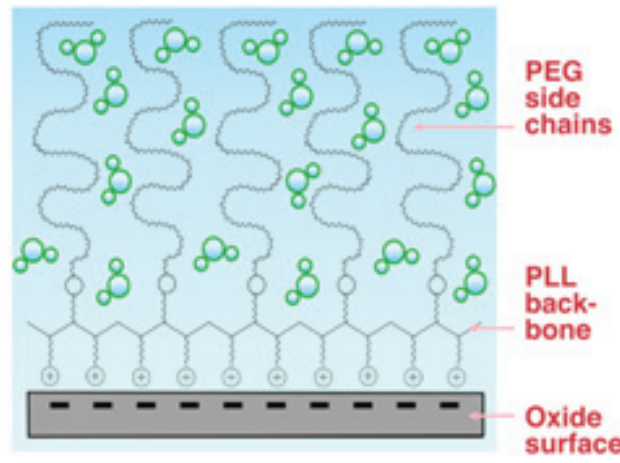

Fig. 3 (a) The structure of poly(L-lysine)-g-poly(ethylene glycol) (PLL-g-PEG). Reprinted from [22] with kind permission from Macromolecules. Copyright 2005, the American Chemical Society. (b) Schematic of PLL-g-PEG adsorption on a negatively charged oxide surface. Reprinted from [23] with kind permission from World Scientific Publishing Company. Copyright 2014

3b). This approach was revolutionary, in that the many surfaces that spontaneously became negatively charged in water could be functionalized with a PEG brush, simply by being dipped into a dilute solution of PLL-g-PEG-a molecule that subsequently became commercially available. Such coatings rendered the surfaces immune to protein adsorption and cell attachment, with the PEG chain density being controllable up to around $0.5 \mathrm{~nm}^{-2}$ (for $2 \mathrm{kDa} P E G$ ) by means of modifying the PEG grafting density on the PLL backbone [21].

\subsubsection{PLL-g-PEG in water or buffer}

In our laboratory, once we had developed experience with PLL- $g$-PEG as a protein-resistance surface, and characterized its behavior extensively with both UHV and liquid-solid interface methods [21,24], it seemed clear that we had to try and use it for lubrication. The initial experiments [25] were carried out under harsh conditions, namely steel-on-steel or steel-on-glass surfaces at loads of up to $5 \mathrm{~N}$ under both rolling and sliding conditions. The inclusion of PLL- $g$-PEG in the lubricating solution led to a roughly $50 \%$ reduction in friction coefficient compared to a control of pure buffer solution, when using PEG side chains of $2 \mathrm{kDa}$ with a lys:PEG ratio of 2.9. Further tribological tests were carried out with a variety of molecular architectures [26], in which the PEG molecular weight, the lys:PEG ratio, and the PLL-backbone molecular weight were all varied. A significant effect of PEG molecular weight was observed, a shift from $2 \mathrm{kDa}$ to $5 \mathrm{kDa}$ resulting in a drop in friction coefficient of an order of magnitude under some conditions (Fig. 4).

Changing lys:PEG ratio also affected the measured friction coefficient under both rolling and sliding conditions, higher PEG densities on the backbone (i.e. lower lys:PEG) translating as higher measured PEG grafting densities on the surface and lower friction coefficients. Backbone length was also found to influence the friction coefficient, with long backbones performing poorly, presumably because of their greater tendency to become partially unbound from the surface and capable of bridging between the sliding partners under low-speed conditions.

While each individual electrostatic interaction of a free amine on the PLL- $g$-PEG backbone with a negative charge on the surface is weak, the effect of many such interactions occurring for each PLL-g-PEG molecule means that spontaneous desorption is rare, although the molecule can apparently be peeled off the surface under tribological conditions. If sliding is taking place in the presence of free PLL-g-PEG, i.e. under a solution, then the peeled-off molecules can readily be replaced, in a "self-healing" mechanism. This could be demonstrated by incorporated two different fluorescent dye molecules into surface-adsorbed and solution-phase PLL-g-PEG, 


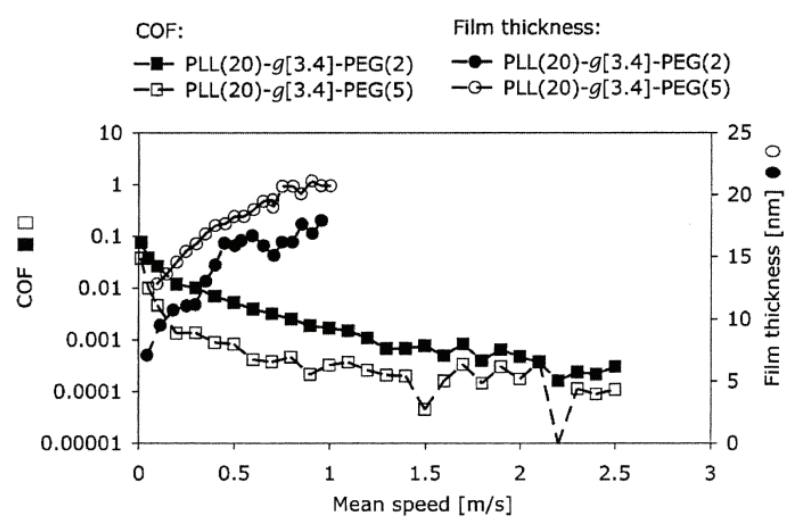

Fig. 4 The influence of the molecular weight of the PEG side chains on the coefficient of friction (squares; $y$-axis on the left-hand-side) and lubricant film thickness (circles; $y$-axis on the right-hand-side) was measured as a function of speed in macroscopic rolling-geometry experiments. The test lubricants were aqueous buffer solutions containing either PLL(20)-g[3.4]-PEG(2) (black symbols) or PLL(20)-g[3.4]-PEG(5) (white symbols). Ball: stainless steel (19 $\mathrm{mm}$ in diameter), substrate:silica, buffer solution: $10 \mathrm{mM}$ HEPES (pH 7.4), polymer concentration. 0.25 $\mathrm{mg} / \mathrm{ml}$, load: $10 \mathrm{~N}$; $\mathrm{T}: 25^{\circ} \mathrm{C}$. Reprinted from [26] with kind permission from Tribology Letters. Copyright 2003, Springer

and carrying out a sliding experiment in a pin-on-disk apparatus [27]. The more sliding cycles that took place, the more replacement of the surface-bound PLL-g-PEG occurred (Fig. 5).

Similar self-healing behavior could also be demonstrated in an experiment with the surface forces apparatus [28], in which friction between mica sheets coated with PLL- $g$-PEG was found to lie below the detection limit of the instrument at low loads, but jumped to high values upon compression to approximately $10 \%$ of the equilibrium brush height, due to abrasion of the polymer from the surface. This abrasion could be significantly mitigated by carrying out the experiment under polymer solution.

Thus it appears that in macroscopic sliding, continuous wear of the lubricating film itself is happening, and this is a dissipative channel that contributes to the friction of the system. One might think that this could be improved by the use of stronger, covalent linkages to the surface. Lee et al. [27] investigated this possibility by comparing the behavior of PLL-g-PEG with that of PEG chains that were individually bonded to the surface via silane linkages. While the behavior of PEG-silane over the first few sliding cycles was very promising, in comparison to a layer of PLL-g-PEG under pure buffer solution, it decayed over a period of some 50 cycles. On the other hand, lubrication under a solution containing PLL- $g$-PEG showed continuously lowered friction, but such a replacement option is not possible for the silane system, since the silane is not stable in aqueous solution.

Under nanotribological conditions, the lower shear forces involved mean that this "wear-dissipation" dissipative channel for PLL-g-PEG can be avoided, and therefore Yan et al. [29] and later Perry et al. [30] investigated the lubricating properties of PLL- $g$-PEG in the atomic force microscope. This was carried out by means of a sodium borosilicate probe sliding against a silicon wafer in a liquid cell. As with the macrotribological studies, PLL- $g$-PEG was found to substantially reduce friction, the lowest frictional values being measured when it was preadsorbed on both sliding surfaces. Also on this measurement scale, increasing the amount of PEG on the surface, by increasing PEG-sidechain molecular weight, by grafting more PEG chains to the backbone, or by incubating the sliding surfaces for a long time prior to the experiment, led to a lower frictional value being measured by lateral force microscopy (LFM). Knowing the polymer architecture, and measuring the adsorbed mass obtained on a silicon dioxide surface, by means of optical waveguide lightmode spectroscopy (OWLS) [31], Perry et al. [30] were able to correlate the friction coefficient, as measured by LFM, with the ratio of PEG-chain spacing and twice the PEG radius of gyration (L/2Rg) (Fig. 6). It can be seen that the friction decreases as $\mathrm{L} / 2 \mathrm{Rg}$ approaches 0.5 , at which point the polymer brush conformation is completely established. A similar trend has been observed for protein adsorption on PEG brushes, which becomes undetectable at $\mathrm{L} / 2 \mathrm{Rg}=0.5$ [21]

While initial aqueous-lubrication studies with PLL- $g$-PEG were carried out with steel, silicon and glass, it was clear that a potential for lubricating ceramic surfaces (often also displaying charged surfaces) existed: It was believed that this could have potential applications, since such ceramics do not show the main problem inherent in aqueous lubrication of steel-corrosion. PLL- $g$-PEG solutions in buffer were tested as aqueous lubricants for silicon carbide and nitride [32] and for silicon carbide were found to be more effective than pure water at all speeds. In the case of silicon nitride, which is readily lubricated by water at high speeds in the absence of polymer, the situation was more complicated, in that the normal mechanism for lubricity in water seemed to be adversely affected by PLL-g-PEG, if this were present during running in. It is during this period that a lubricious, ultrasmooth layer of hydroxylated material is believed to form, which later facilitates hydrodynamic lubrication [33-35]. However, if the polymer is added after running in, it apparently adsorbs on the outer layer and enables silicon nitride to display low friction values in both lowand high-speed regimes.

From the structure of PLL-g-PEG (Fig. 3a), it can be 


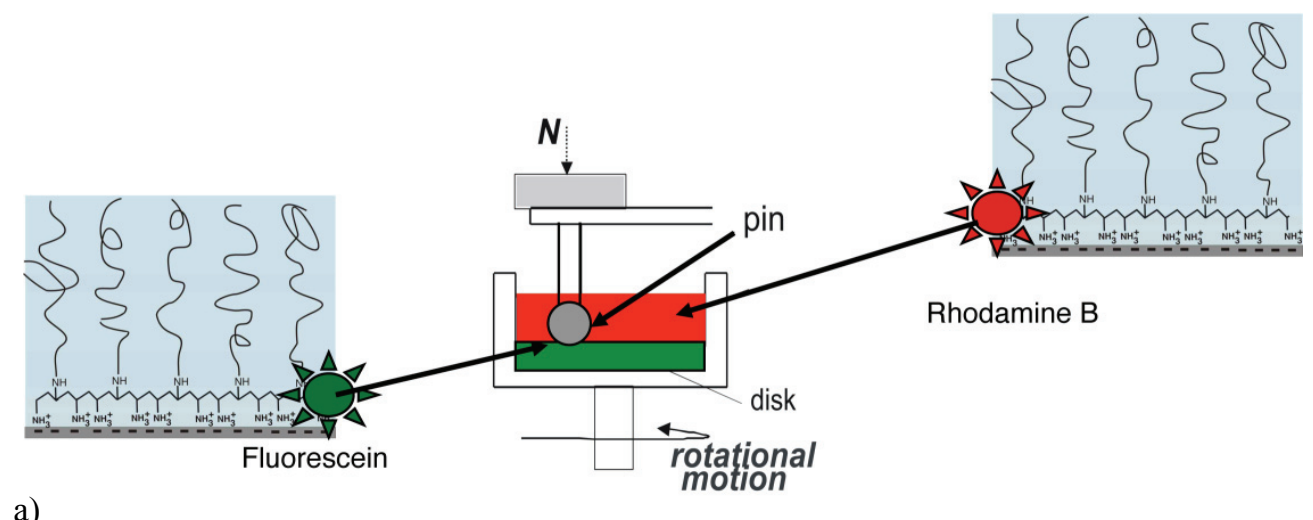

a)

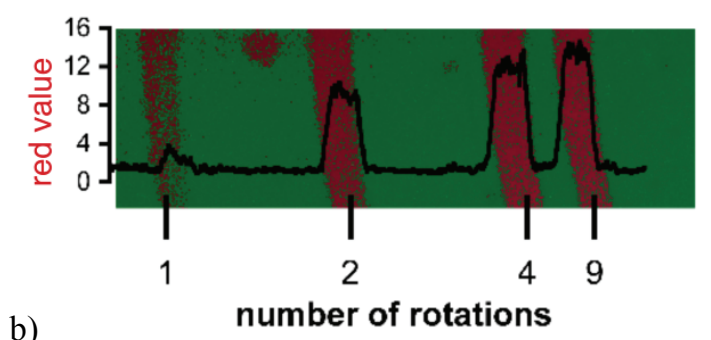

Fig. 5 (a):Experimental setup to determine the extent to which surface-bound PLL- $g$-PEG is being replaced by PLL- $g$-PEG in solution by means of fluorescence microscopy, (b) Image from the setup in 5(a), showing the increased degree of replacement of surface PLL-g-PEG by that in the solution, after sliding at 5 $\mathrm{mm} / \mathrm{s}, 2 \mathrm{~N}$ load (steel on glass). Reprinted from [27] with kind permission from Tribology Letters. Copyright 2006, Springer

seen that the lysine units incorporate a four- $\mathrm{CH}_{2}$-group chain between the peptide backbone and the amine group. This has two interesting consequences. First of all, the extra flexibility that this imparts to the location of the amine group on the surface apparently allows for optimization of the relative positions of charged amine and surface charge, leading to an overall stronger interaction with the surface. This could be investigated by substituting poly(allyl amine) (PAAm) for poly(L-lysine) in the polymer [36]. PAAm consists of a hydrocarbon backbone, with amines joined to every other carbon via a single $\mathrm{CH}_{2}$-group only. While PAAm- $g$-PEG was found to lubricate as well as PLL-g-PEG under macroscopic rolling conditions, in sliding geometry, in which shear forces are much greater, the relative performance of PAAm- $g$-PEG was considerably reduced. Furthermore, adsorption measurements showed that irrespective of grafting ratio, for similar backbone lengths the surface PEG density was lower for PAAm- $g$-PEG than for PLL- $g$-PEG.

Another interesting consequence of the four $\mathrm{CH}_{2}$-groups (i.e. an n-butyl chain) is the fact that PLL-g-PEG is able to adsorb on and lubricate hydrophobic surfaces. This has the major consequence that PLL-g-PEG is effectively able to lubricate all three materials classes. Although the interaction of the individual n-butyl chain is weak, the combination of over 1000 of them that are available on a single PLL chain in PLL(375 kDa)- $g$ [3.4]-PEG(5 kDa) becomes significant. In a comparative study with pluronic adsorption on thermoplastics, Lee and Spencer [37] showed that PLL(375)- $g$ [3.4]-PEG(5) adsorbed on PDMS with about $50 \%$ of the mass uptake of the pluronic $\mathrm{EO}_{37}-\mathrm{PO}_{56}-\mathrm{EO}_{37}$. In terms of lubrication, while in self-mated sliding of PE, $\mathrm{PP}$, and polyamide $(6,6)$ in water, the pluronic lubricated slightly better than the PLL- $g$-PEG, when the thermoplastics were slid against glass, steel or $\mathrm{ZrO}_{2}$ ceramic, the PLL-g-PEG came into its own, lubricating the system very effectively since it was able to adsorb on both surfaces, thereby providing brush-brush contact. Tuning of the solution parameters appears to be critical, however, for PLL-g-PEG lubrication of hydrophobic systems. The adsorption degree and therefore the lubricating efficiency, are highly influenced by factors such as $\mathrm{pH}$ and ionic strength [38].

If the polymers are initially plasma treated, prior to PLL- $g$-PEG adsorption, thereby acquiring a negative charge, adsorption of and lubrication by the positively charged PLL-g-PEG becomes particularly effective. Untreated PDMS sliding against PDMS in pure water shows very high friction, due to the large component of hydrophobic interaction. However, upon plasma 


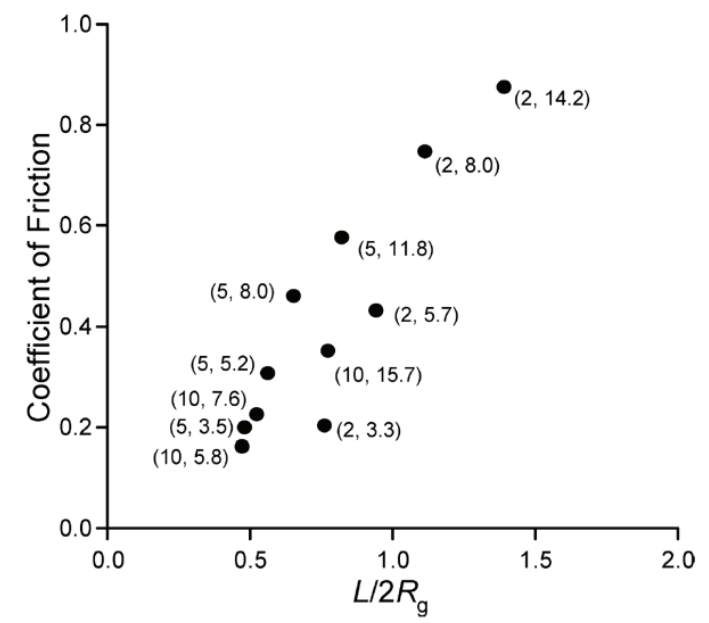

Fig. 6 Plot of the coefficient of friction versus $\mathrm{L} / 2 \mathrm{Rg}$ for a series of PLL-g-PEG samples synthesized with different architectures, where $\mathrm{L}$ is the spacing between PEG chains and $\mathrm{Rg}$ is the radius of gyration of the PEG chains. The friction was measured by lateral force microscopy with a bare sodium borosilicate colloidal probe. The labels $(z, y)$ indicate the PEG molecular weight in $\mathrm{kDa}(z)$ and the lys/PEG grafting ratio (y). Reprinted from [30] with kind permission from ACS Applied Materials and Interfaces. Copyright 2009, the American Chemical Society

treatment, this interaction disappears, since the PDMS becomes hydrophilic, and far lower friction is observed, with a slight upward trend at lower speeds, due to the onset of the boundary regime. Upon PLL- $g$-PEG adsorption, the higher-speed data shows the same friction coefficient, but apparently the onset of boundary lubrication is eliminated, since the solid surfaces are held apart by lubricious brushes [39] (Fig. 7).

Natural materials often contain surface charge, and this was the original motivation for Elbert and Hubbell's work with PLL-g-PEG [19]. An example of a charged natural material that we expose to an aqueous environment on a daily basis is hair, and the interaction of PLL-g-PEG with hair was investigated by Lee et al. [41]. By incorporating a fluorescent dye into the PLL-g-PEG, it could be observed, by fluorescence microscopy, that it adsorbed unevenly on bleached hair, presumably due to the uneven charge distribution, but was nevertheless highly effective at lubrication, significantly reducing friction coefficient, as measured by LFM. Cationic surfactants, as commonly used in the cosmetics industry, were found to remove the PLL-g-PEG from the surface and led to a restoration of the original friction values, prior to PLL-g-PEG treatment.

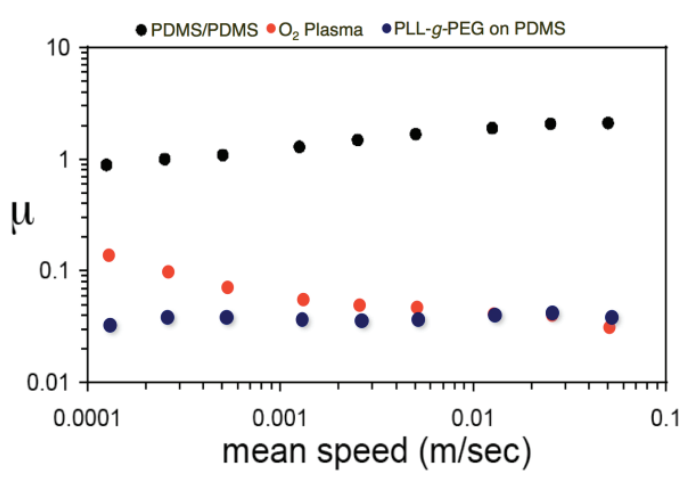

Fig. 7 PDMS pin sliding against PDMS disk, $1 \mathrm{~N}$ load, in water, untreated (black dots), plasma treated (red dots), and in the presence of PLL-g-PEG (blue dots). While soft elastohydrodynamic lubrication appears to reduce friction once the PDMS has been hydrophlized by the plasma treatment, the PLL-g-PEG lubricates better still at low speeds due to the elimination of the boundary regime and its replacement by brush lubrication. Adapted from [40] with kind permission from Langmuir. Copyright 2005, the American Chemical Society

\subsubsection{PLL- $g$-PEG in other solvent environments}

Water is a good solvent for PEG, and therefore PEG brushes in water appear to provide more brush-like chain configuration, and lower friction coefficients than PEG brushes in less-good solvents. Müller et al. [22] showed by means of LFM, OWLS, and the quartz-crystal microbalance, that upon examining the solvent series, water, methanol, thanol, 2-propanol, the amount of solvent in the brush steadily decreases. They also found this quantity to correlate inversely with the measured friction. In a mixed-solvent system, the same authors were able to show that if one component of the mixture is a good solvent (aqueous buffer) and one not (2-propanol), the water resides preferentially within the brush as the concentration of 2-propanol is increased. The friction therefore remains essentially constant until the volume fraction of 2-propanol exceeds 0.8 [42].

While aqueous PLL-g-PEG solution appears to be effective in lubricating many different surfaces at low speeds, its very low viscosity means that hydrodynamic lubrication is only possible at high speeds. One solution to this problem is to add another, more viscous component to the solvent. Nalam et al. used glycerol and ethylene glycol to this end [43], mixing them in various concentrations into water, and using the mixture to lubricate steel against glass under macroscopic sliding and rolling conditions. The effects of the glycerol and the PEG brushes seem to be largely independent and additive, as shown in Fig. 8 for macroscopic, 


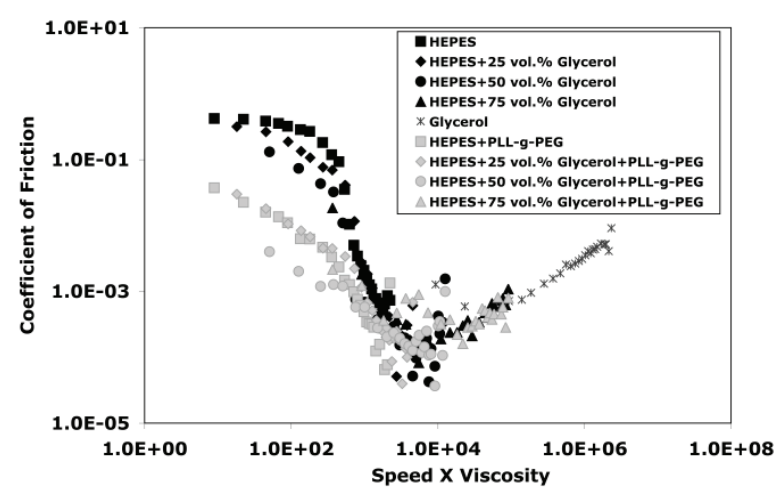

Fig. 8 (Speed $\times$ viscosity) dependence of coefficient of friction $(\mu)$ from mini-traction-machine (MTM) measurements (Steel on glass, $10 \mathrm{~N}$ ) for different buffer-glycerol mixtures, with and without PLL-g-PEG. Reprinted from [43] with kind permission from Tribology Letters. Copyright 2010, Springer

steel-ball-on-glass-disk rolling experiments. The effect of the greater viscosity of glycerol-containing buffer solution (HEPES) leads to a shift to the right on the (same) Stribeck curve for a given combination of load and speed, while the addition of the PLL-g-PEG clearly lowers the friction measured in the boundary-lubrication regime.

While much of the reported work on PLL-g-PEG has involved either very smooth, model surfaces, or technical surfaces with an intrinsic roughness, little has been done to explore the interplay of roughness and brush lubrication. One exception is a study of Ramakrishna et al. [44], who used a roughness gradient, fabricated by firmly attaching 12-nm silica nanospheres onto a silicon surface. The friction was measured by LFM, using a polyethylene colloidal probe. In the absence of adsorbed PLL- $g$-PEG, the measured friction is dominated by adhesion, which is maximum at the smooth end of the gradient (no particles), and minimum at the rough end. Following polymer adsorption, there is no measurable adhesion, and the friction, which is greatly reduced, shows an opposite trend, increasing gradually from the smooth end to the rough end. Although infrared spectroscopy showed that more polymer was adsorbed at the rough end, as might be expected from its slightly increased specific surface area, it was concluded that the high curvature of the silica particles (with a diameter comparable to the brush length) led to a reduction in PEG swelling compared to PEG adsorbed on a flat substrate, and that this led to a concomitant reduction in lubrication ability (Fig. 9).

\subsection{Other means of PEG surface attachment}

Several alternative block-copolymer-based approaches have been used to anchor PEG on surfaces for lubrication

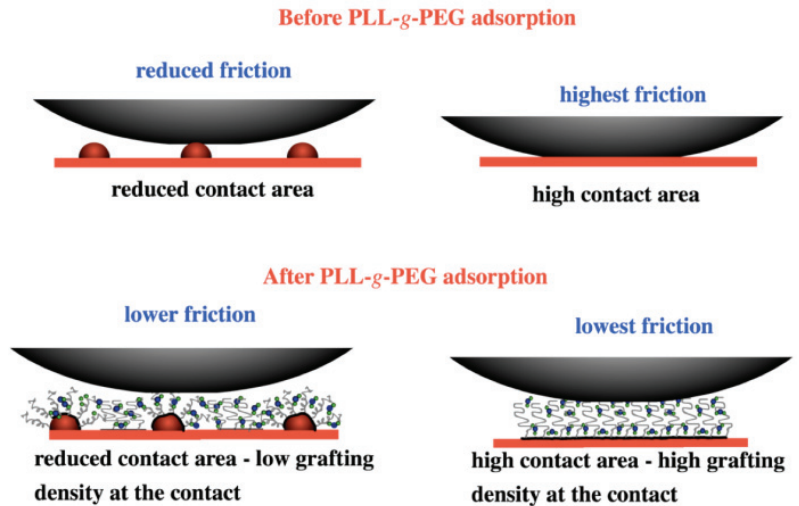

Fig. 9 Effect of controlled, nanoscale roughness on lubricating ability of PEG brushes. Reprinted from [44] with kind permission from Langmuir. Copyright 2013, the American Chemical Society

in addition to the PEO-PPO blocks described in section 2. Most have these have, like PLL- $g$-PEG, involved charged blocks, such as the PAAm- $g$-PEG case mentioned briefly above, in section 3.1. PAAm- $g$-PEG lubricates in general less well than PLL-g-PEG for the reasons described above, but has the single advantage of being considerably cheaper to produce!

Brady et al. [45] synthesized poly(ethyleneimine)-g-poly (ethylene glycol) (PEI-g-PEG) using branched PEI, and found it to have lubricating properties that very much resembled those of PLL-g-PEG, despite the very different molecular architecture, due to the use of a branched backbone.

Pettersson et al. [46] copolymerized $\mathrm{MeO}-(\mathrm{EG})_{45^{-}}$ and $\mathrm{Me}_{3} \mathrm{~N}^{+}-\mathrm{C}_{2} \mathrm{H}_{4}$-methacrylates, thereby producing a bottle-brush polymer, where some sidechains were $\operatorname{PEG}(2 \mathrm{kDa})$ and some were quaternary amines, bearing a positive charge. In other words, quite a similar structure to PLL-g-PEG. Lubrication properties of these polymers showed a dependence on the PEG:amine ratio, as might be expected, but surprisingly the lowest friction was obtained for a polymer architecture, in which only $10 \%$ of the monomers consisted of the charged anchoring groups. This is a PEG density on a backbone that could not be achieved by the PLL- $g$-PEG route, and it is interesting to note that it is apparently in a regime where the PEG becomes involved in bonding to the surface, and is outside of the normal polymer-brush regime. Another, more promising, approach from the same laboratory [47], using the same monomers, was to create a diblock copolymer of poly (MeO-(EG) $)_{45}$-methacrylate) and poly $\left(\mathrm{Me}_{3} \mathrm{~N}^{+}-\mathrm{C}_{2} \mathrm{H}_{4}\right.$-methacrylate), such that the cationic tail firmly anchored the PEG(2000)-sidechain-containing, neutral bottlebrush to the surface, to produce, at maximum coverage, a highly hydrated layer, $48 \mathrm{~nm}$ in thickness. This layer provided a lubricious surface that, 


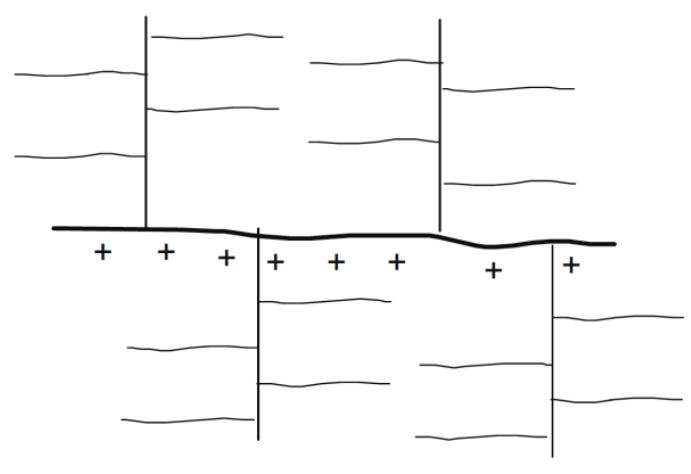

Fig. 10 Schematic representation of the polymer architecture of the brush-on-brush polyelectrolytes AETAC-X-graft-PEO ${ }_{45}$ MEMA. Reprinted from [49] by kind permission from European Polymer Journal. Copyright 2010, Elsevier

providing it had been adsorbed in low-ionic-strength conditions, showed considerable resistance to desorption in high-salt conditions, albeit with a reduced lubricating capability [48]. Extending this approach still further, the same group has synthesized "AETAC-X-graft-PEO ${ }_{45}$ MEMA" comb polymers, where the side-chains consist of PEG(2000) chains on a methacrylate backbone, grown by atom-transfer radical polymerization from a poly (acryloxyethyl trimethylammonium chloride) backbone [49]. A schematic of the structure is shown in Fig. 10.

This polymer appears to adsorb well on silica surfaces, and displayed friction coefficients measured by colloidal-probe LFM of as low as 0.02 for silica-mica sliding (both sides coated) in water.

In quite a different approach, Ding et al. [50] catalytically incorporated PEG-methacrylate (300 Da) into hydrosiloxane-blocked PDMS (one component of the commercial PDMS kit, Sylgard 184, Dow-Corning) before crosslinking it with vinylsiloxane-blocked PDMS (the other component of Sylgard 184). The result was PDMS with grafted PEG, which showed greater hydrophilicity and water-lubricity than normal PDMS.

A number of alternative ways have been suggested for the covalent attachment of PEG to surfaces, including the silane approach described above [27]. In a study of the effect of ionic strength on PEG brushes themselves, Heeb et al. [51] studied the lubrication of gold surfaces with PEG (5000)-thiol. They found that high ionic strength was deleterious to the lubrication performance of the PEG, presumably due to salting-out effect on the PEG, leading to its reduced hydration and partial collapse. Macroscopic frictional measurements were carried out on these thiol-anchored polymer chains by using a soft countersurface of oxidized PDMS.

L-3,4-dihydroxyphenylalanine)-lysine (DOPA-K) polymerizes on surfaces under basic conditions, bonding to them by hydrophobic or coordinative interactions, depending on the nature of the substrate. This is the basis of the attachment mechanism used by mussels to anchor themselves to rocks [52]. By using PEG(5 kDa)-DOPA-K, Chawla et al. [53] were able to coat PDMS with PEG, and impart it with considerable lubricity, demonstrating friction coefficients between self-mated, PEG-DOPA-K-treated PDMS of 0.03 at $1 \mathrm{~N}$ load.

\section{Aqueous lubrication: beyond PEG brushes}

As stated at the beginning, while PEG brushes have served well as a model system, true applications require hydrophilic polymer brushes that are more stable in the long term. One attempt to replace PEG, by Perrino et al., was to prepare a PLL- $g$-PEG analog with dextran instead of PEG [54]. While this polymer does indeed lubricate almost as well as PLL-g-PEG under some conditions, can be synthesized more cheaply than PLL-g-PEG, and is more resistant to long-term oxidation, it has a fatal flaw, in that it is highly susceptible to bacterial attack [55].

Grafting-from approaches to covering surfaces with hydrated polymer brushes have been used increasingly in recent years. Heeb et al. [56] grew poly(methacrylic acid) layers to some hundreds of nm dry thickness. These layers swelled significantly in water and showed very low friction $(\mu<0.01)$ when slid against oxidized PDMS at a load of $1 \mathrm{~N}$.

An interesting approach to lubricious brushes has been described by Chen et al. [2,57], who used a copolymer of a quaternary amine-containing methacrylate and a methacrylate containing an ATRP initiator to grow chains of the biomimetic and biocompatible polyzwitterion, poly(2-(methacryloyloxy)ethyl phosphorylcholine), (poly(MPC)) from a mica surface. These electrostatically anchored polymer chains exhibited extremely low friction $(\mu \approx 0.001)$ in the surface forces apparatus, at pressures up to $7.5 \mathrm{MPa}$. Yamada et al. looked at similar poly(MPC) brushes on a glass substrate under macrotribological conditions $(0.49 \mathrm{~N})$, and measured a friction coefficient in water against a poly(MPC)-covered glass ball of around $0.1 \mathrm{~N}$ [58]. Surprisingly, under air of $80 \% \mathrm{RH}$, this value reduced to 0.02. The authors attributed this to a reduction of interdigitation in humid air, compared to that in water.

\section{Conclusions}

Many important properties of PEG brushes have been studied over recent years, and these have led to a greater awareness of the behavior of hydrophilic brushes in general. This period is drawing to a close. The next step is to apply such knowledge to the design of new generations of aqueous lubricants, which not only display lubricity, but also long-term stability with respect to oxidation and tribological stress. Such lubricants have the potential to reduce the consumption of oil-based 
additives, and to lead to an overall saving of energy in sliding and rolling systems - especially those operating at low speeds.

\section{References}

[1] Lee, S. and Spencer, N. D., "Materials Science Sweet, Hairy, Soft, and Slippery," Science, 319, 5863, 2008, 575-576.

[2] Chen, M., Briscoe, W. H., Armes, S. P. and Klein J., "Lubrication at Physiological Pressures by Polyzwitterionic Brushes," Science, 323, 5922, 2009, 1698-1701.

[3] Banquy, X., Burdyńska, J., Lee, D. W., Matyjaszewski, K. and Israelachvili, J., "Bioinspired Bottle-Brush Polymer Exhibits Low Friction and Amontons-like Behavior," J. Am. Chem. Soc., 136, 17, 2014, 6199-6202.

[4] Gohar, R., "Elastohydrodynamics," World Scientific Publishing Company, Singapore, 2001.

[5] Schmelzer, J. W. P., Zanotto, E. D. and Fokin, V. M., "Pressure Dependence of Viscosity," J. Chem. Phys., 122, 7, 2005, 074511.

[6] Lee, S., "Sticky and Slippery: Interfacial Forces of Mucin and Mucus Gels," In: Spencer, N.D., editor. "Aqueous Lubrication," World Scientific Publishing Company, Singapore, 2014, 33.

[7] Milner, S., "Polymer Brushes," Science, 251, 4996, 1991, 905-914.

[8] Klein, J., Kumacheva, E., Mahalu, D., Perahia, D. and Fetters, L., "Reduction of Frictional Forces between Solid-Surfaces Bearing Polymer Brushes," Nature, 370, 6491, 1994, 634-636.

[9] Klein, J., "Shear, Friction, and Lubrication Forces between Polymer-Bearing Surfaces," Annu. Rev. Mater. Sci., 26, 1, 1996, 581-612.

[10] Raviv, U., Tadmor, R. and Klein, J., "Shear and Frictional Interactions between Adsorbed Polymer Layers in a Good Solvent," J. Phys. Chem. B, 105, 34, 2001, 8125-8134.

[11] Espinosa-Marzal, R. M., Fontani, G., Reusch, F. B., Roba, M., Spencer, N. D. and Crockett, R., "Sugars Communicate through Water: Oriented Glycans Induce Water Structuring," Biophys. J., 104, 12, 2013, 2686-2694.

[12] Leckband, D., Sheth, S. and Halperin, A., "Grafted Poly(Ethylene Oxide) Brushes as Nonfouling Surface Coatings," J. Biomater. Sci. Polym. Ed, 10, 10, 1999, 1125-1147.

[13] Branch, D. W., Wheeler, B. C., Brewer, G. J. and Leckband, D. E., "Long-Term Stability of Grafted Polyethylene Glycol Surfaces for Use with Microstamped Substrates in Neuronal Cell Culture," Biomaterials, 22, 10, 2001, 1035-1047.

[14] Saeki, S., Kuwahara, N., Nakata, M. and Kaneko, M., "Upper and Lower Critical Solution Temperatures in Poly (ethylene glycol) Solutions," Polymer, 17, 8, 1976, 685-689.
[15] Raviv, U., Frey, J., Sak, R., Laurat, P., Tadmor, R. and Klein, J., "Properties and Interactions of Physigrafted End-Functionalized Poly(Ethylene Glycol) Layers," Langmuir, 18, 20, 2002, 7482-7495.

[16] Chai, L. and Klein, J., "Shear Behavior of Adsorbed Poly(Ethylene Oxide) Layers in Aqueous Media," Macromolecules, 41, 5, 2008, 1831-1838.

[17] Lee, S., Iten, R., Müller, M. and Spencer, N. D., "Influence of Molecular Architecture on the Adsorption of Poly(Ethylene Oxide)-Poly (Propylene Oxide)-Poly(Ethylene Oxide) on PDMS Surfaces and Implications for Aqueous Lubrication," Macromolecules, 37, 22, 2004, 8349-8356.

[18] Li, Y., Rojas, O. J. and Hinestroza, J. P., "Boundary Lubrication of PEO-PPO-PEO Triblock Copolymer Physisorbed on Polypropylene, Polyethylene, and Cellulose Surfaces," Ind. Eng. Chem. Res., 51, 7, 2012, 2931-2940.

[19] Elbert, D. L. and Hubbell J. A., "Self-Assembly and Steric Stabilization at Heterogeneous, Biological Surfaces using Adsorbing Block Copolymers," Chemistry \& Biology, 5, 3, 1998, 177-183.

[20] Kenausis, G. L., Vörös, J., Elbert, D. L., Huang, N.-P., Hofer, R., Ruiz-Taylor, L., Textor, M., Hubbell, J. A. and Spencer, N. D., "Poly(L-Lysine)-g-Poly(Ethylene Glycol) Layers on Metal Oxide Surfaces: Attachment Mechanism and Effects of Polymer Architecture on Resistance to Protein Adsorption," J. Phys. Chem. B, 104, 14, 2000, 3298-3309.

[21] Pasche, S., De Paul, S. M., Vörös, J., Spencer, N. D. and Textor, M., "Poly(L-Lysine)-graft-Poly(Ethylene Glycol) Assembled Monolayers on Niobium Oxide Surfaces: A Quantitative Study of the Influence of Polymer Interfacial Architecture on Resistance to Protein Adsorption by ToF-SIMS and in Situ OWLS," Langmuir, 19, 22, 2003, 9216-9225.

[22] Müller, M. T., Yan, X., Lee, S., Perry, S. S. and Spencer N. D., "Lubrication Properties of a Brushlike Copolymer as a Function of the Amount of Solvent Absorbed within the Brush," Macromolecules, 38, 13, 2005, 5706-5713.

[23] Giasson, S. and Spencer, N. D. "Aqueous Lubrication with Polymer Brushes," In: Spencer N.D., editor. "Aqueous Lubrication," World Scientific Publishing Company, Singapore, 2014, 183.

[24] Huang, N. P., Michel, R. Vörös, J., Textor, M., Hofer, R., Rossi, A. Elbert, D. L., Hubbell, J. A. and Spencer, N. D., "Poly(L-Lysine)-g-Poly (Ethylene Glycol) Layers on Metal Oxide Surfaces: Surface Analytical Characterization and 
Resistance to Serum and Fibrinogen Adsorption," Langmuir, 17, 2, 2001, 489-498.

[25] Lee, S., Müller, M., Ratoi-Salagean, M., Vörös, J., Pasche, S., De Paul, S. M., Spikes, H. A., Textor, M. and Spencer, N. D., "Boundary Lubrication of Oxide Surfaces by Poly(L-Lysine)-g-Poly(Ethylene Glycol) (PLL-g-PEG) in Aqueous Media," Tribol. Lett., 15, 3, 2003, 231-239.

[26] Müller, M., Lee, S., Spikes, H. A. and Spencer, N. D., "The Influence of Molecular Architecture on the Macroscopic Lubrication Properties of the Brush-Like Co-Polyelectrolyte Poly(L-Lysine) -g-Poly(Ethylene Glycol) (PLL-g-PEG) Adsorbed on Oxide Surfaces," Tribol. Lett., 15, 4, 2003, 395-405.

[27] Lee, S., Müller, M., Heeb, R., Zuercher, S., Tosatti, S., Heinrich, M., Amstad, F., Pechmann, S. and Spencer, N. D., "Self-Healing Behavior of a Polyelectrolyte-Based Lubricant Additive for Aqueous Lubrication of Oxide Materials," Tribol. Lett., 24, 3, 2006, 217-223.

[28] Drobek, T. and Spencer, N. D., "Nanotribology of Surface-grafted PEG Layers in an Aqueous Environment," Langmuir, 24, 4, 2008, 1484-1488.

[29] Yan, X., Perry, S. S., Spencer, N. D., Pasche, S., De Paul, S. M., Textor, M. and Lim, M. S., "Reduction of Friction at Oxide Interfaces upon Polymer Adsorption from Aqueous Solutions," Langmuir, 20, 2, 2004, 423-428.

[30] Perry, S. S., Yan, X., Limpoco, F. T., Lee, S., Müller, M. and Spencer, N. D., "Tribological Properties of Poly(L-Lysine)-graft-Poly(Ethylene Glycol) Films: Influence of Polymer Architecture and Adsorbed Conformation," ACS Appl. Mater. Inter., 1, 6, 2009, 1224-1230.

[31] Vörös, J., Ramsden, J. J., Csucs, G., Szendro, I., De Paul, S. M., Textor, M. and Spencer, N. D., "Optical Grating Coupler Biosensors," Biomaterials, 23, 17, 2002, 3699-3710.

[32] Hartung, W., Rossi, A., Lee, S. and Spencer, N. D., "Aqueous Lubrication of $\mathrm{SiC}$ and $\mathrm{Si}_{3} \mathrm{~N}_{4}$ Ceramics Aided by a Brush-Like Copolymer Additive, Poly(L-Lysine)-graft-Poly(Ethylene Glycol)," Tribol. Lett., 34, 3, 2009, 201-210.

[33] Wong, H.-C., Umehara, N. and Kato, K., "The Effect of Surface Roughness on Friction of Ceramics Sliding in Water," Wear, 218, 2, 1998, 237-243.

[34] Tomizawa, H. and Fischer, T. E., "Friction and Wear of Silicon Nitride and Silicon Carbide in Water: Hydrodynamic Lubrication at Low Sliding Speed Obtained by Tribochemical Wear," ASLE Transactions, 30, 1, 1987, 41-46.

[35] Muratov, V. A. and Fischer, T. E., "Tribochemical Polishing," Annu. Rev. Mater. Sci., 30, 1, 2000, 27-51.
[36] Hartung, W., Drobek, T., Lee, S., Zürcher, S. and Spencer, N. D., "The Influence of Anchoring-Group Structure on the Lubricating Properties of Brush-Forming Graft Copolymers in an Aqueous Medium," Tribol. Lett., 2008, 31, 2, 119-128.

[37] Lee, S. and Spencer, N. D., "Poly(L-Lysine)-graft-Poly(Ethylene Glycol): a Versatile Aqueous Lubricant Additive for Tribosystems Involving Thermoplastics," Lubrication Science, 20, 1, 2008, 21-34.

[38] Lee, S. and Spencer, N. D., "Adsorption Properties of Poly(L-Lysine)-graft-Poly(Ethylene Glycol) (PLL-g-PEG) at a Hydrophobic Interface: Influence of Tribological Stress, $\mathrm{pH}$, Salt Concentration, and Polymer Molecular Weight," Langmuir, 24, 17, 2008, 9479-9488.

[39] Lee, S. and Spencer, N. D., "Aqueous Lubrication of Polymers: Influence of Surface Modification," Tribol. Int., 38, 11-12, 2005, 922-930.

[40] Lee, S. and Vörös, J., “An Aqueous-Based Surface Modification of Poly(Dimethylsiloxane) with Poly(Ethylene Glycol) to Prevent Biofouling," Langmuir, 21, 25, 2005, 11957-11962.

[41] Lee, S., Zürcher, S., Dorcier, A., Luengo, G. S. and Spencer, N. D., "Adsorption and Lubricating Properties of Poly(L-Lysine)-graft-Poly(Ethylene Glycol) on Human-Hair Surfaces," ACS Appl. Mater. Inter., 1, 9, 2009, 1938-1945.

[42] Müller, M. T., Yan, X., Lee, S., Perry, S. S. and Spencer, N. D., "Preferential Solvation and its Effect on the Lubrication Properties of a Surface-Bound, Brushlike Copolymer," Macromolecules, 38, 9, 2005, 3861-3866.

[43] Nalam, P. C., Clasohm, J. N., Mashaghi, A. and Spencer, N. D., "Macrotribological Studies of Poly(L-Lysine)-graft-Poly(Ethylene Glycol) in Aqueous Glycerol Mixtures," Tribol. Lett., 37, 3, 2010, 541-552.

[44] Ramakrishna, S. N., Espinosa-Marzal, R. M., Naik, V. V., Nalam, P. C. and Spencer, N. D., "Adhesion and Friction Properties of Polymer Brushes on Rough Surfaces: a Gradient Approach," Langmuir, 29, 49, 2013, 15251-15259.

[45] Brady, M. A., Limpoco, F. T. and Perry, S. S., "Solvent-Dependent Friction Force Response of Poly(Ethylenimine)-graft-Poly(Ethylene Glycol) Brushes Investigated by Atomic Force Microscopy," Langmuir, 25, 13, 2009, 7443-7449.

[46] Pettersson, T., Naderi, A., Makuska, R. and Claesson, P. M., "Lubrication Properties of Bottle-Brush Polyelectrolytes: An AFM Study on the Effect of Side Chain and Charge Density," Langmuir, 24, 7, 2008, 3336-3347.

[47] Liu, X., Dėdinaite, A., Rutland, M., Thormann, E., Visnevskij, C., Makuska, R. and Claesson, P. M., "Electrostatically Anchored Branched Brush Layers," Langmuir, 28, 44, 2012, 15537-15547. 
[48] Liu, X., Thormann, E., Dėdinaitè, A., Rutland, M., Visnevskij, C., Makuska, R. and Claesson, P. M., "Low Friction and High Load Bearing Capacity Layers Formed by Cationic- Block -Non-Ionic Bottle-Brush Copolymers in Aqueous Media," Soft Matter, 9, 22, 2013, 5361-5371.

[49] Krivorotova, T., Makuska, R., Naderi, A., Claesson, P. M. and Dedinaite, A., "Synthesis and Interfacial Properties of Novel Cationic Polyelectrolytes with Brush-on-Brush Structure of Poly (Ethylene Oxide) Side Chains," European Polymer Journal, 46, 2, 2010, 171-180.

[50] Ding, Y., Jiao, Z.-S., Guo, D.-J., Xiao, S.-J., Tan, W. and Dai, Z.-D., "Tunable Cohesion and Water Lubrication of PEG-g-PMHS-c-PMVS Copolymer Membranes," Colloid Surface A, 395, 2012, 199-206.

[51] Heeb, R., Lee, S., Venkataraman, N. V. and Spencer, N. D., "Influence of Salt on the Aqueous Lubrication Properties of End-grafted, Ethylene Glycol-Based Self-Assembled Monolayers," ACS Appl. Mater. Inter., 1, 5, 2009, 1105-1112.

[52] Brubaker, C. E. and Messersmith, P. B., "The Present and Future of Biologically Inspired Adhesive Interfaces and Materials," Langmuir, 28, 4, 2012, 2200-2205.

[53] Chawla, K., Lee, S., Lee, B. P., Dalsin, J. L., Messersmith, P. B. and Spencer, N. D., "A Novel Low-Friction Surface for Biomedical Applications: Modification of Poly(Dimethylsiloxane) (PDMS)
Biomed. Mater. Res. A, 90, 3, 2009, 742-749.

[54] Perrino, C., Lee, S. and Spencer, N. D., "End-grafted Sugar Chains as Aqueous Lubricant Additives: Synthesis and Macrotribological Tests of Poly(L-Lysine)-graft-Dextran (PLL-g-Dex) Copolymers," Tribol. Lett., 33, 2, 2009, 83-96.

[55] Argibay, N., Perrino, C., Rimann, M., Lee, S. and Spencer, N. D., "Bacterially Induced Degradation of Aqueous Solutions of Poly(L-Lysine)-graft-Poly(Ethylene Glycol) and Poly(L-Lysine)-graft-Dextran: Consequences for their Lubrication Properties," Lubrication Science, 21, 10, 2009, 415-425.

[56] Heeb, R., Bielecki, R. M., Lee, S. and Spencer, N. D., "Room-Temperature, Aqueous-Phase Fabrication of Poly(Methacrylic Acid) Brushes by UV-LED-Induced, Controlled Radical Polymerization with High Selectivity for Surface-Bound Species," Macromolecules, 42, 22, 2009, 9124-9132.

[57] Chen, M., Briscoe, W. H., Armes, S. P., Cohen, H. and Klein, J., "Robust, Biomimetic Polymer Brush Layers Grown Directly from a Planar Mica Surface," Chemphyschem, 8, 9, 2007, 1303-1306.

[58] Kobayashi, M., Terayama, Y., Hosaka, N., Kaido, M., Suzuki, A., Yamada, N., Torikai, N., Ishihara, K. and Takahara, A., "Friction Behavior of High-Density Poly(2-methacryloyloxyethyl phosphorylcholine) Brush in Aqueous Media," Soft Matter, 3, 6, 2007, 740-746. 